\title{
SARCOPENIA E FATORES ASSOCIADOS EM PACIENTES COM DOENÇA RENAL CRÔNICA EM TRATAMENTO DIALÍTICO PERITONEAL
}

\section{Nayara Cristine Penha Rosa}

Nutricionista. Universidade Federal do Espírito Santo. Departamento de Educação em Saúde (DEIS). Curso de nutrição, Brasil.

\section{Fernanda Godinho de Alcântara}

Nutricionista. Universidade Federal do Espírito Santo (UFES). Departamento de Educação em Saúde (DEIS). Curso de nutrição, Brasil.

\section{Monica Cattafesta}

Mestra em Nutrição e Saúde. Universidade Federal do Espírito Santo (UFES). Programa de Pós-graduação em Saúde Coletiva (PPGSC), Brasil.

\section{Aparecida Ferreira Furriel}

Nutricionista. Hospital Universitário Cassiano Antônio de Moraes (HUCAM), Brasil.

\section{Luciane Bresciani Salaroli}

Doutora em Ciências Fisiológicas. Programa de Pós-graduação em Saúde Coletiva, Programa de Pós-graduação em Nutrição e Saúde, Brasil.

\begin{abstract}
RESUMO: Avaliar a presença de sarcopenia e fatores associados em pacientes com Doença Renal Crônica (DRC) que realizaram diálise peritoneal (DP) em ambulatório de nefrologia de um hospital universitário. Trata-se de estudo transversal com usuários do serviço de DP de um hospital universitário, de ambos os sexos. Foram coletados dados sociodemográficos, das condições clínicas, hemodinâmicos e antropométricos. Foram coletados dados de $100 \%(\mathrm{n}=35)$ dos indivíduos em DP do serviço estudado. A sarcopenia foi detectada em 100\% (n $=14$ ) dos homens. Os desnutridos, segundo área muscular do braço corrigida e perímetro do braço, $78,6 \%(n=11)$ e $71,4 \%(n=10)$ eram sarcopênicos, assim como a maioria dos hipertensos $57,1 \%$, $(n=8)$. A sarcopenia também se associou positivamente à hipertrigliceridemia. Fatores como sexo, raça/cor, doenças de base secundárias a comorbidades, pressão arterial e parâmetros de estado nutricional tiveram associação com a presença de sarcopenia na população estudada.
\end{abstract}

PALAVRAS-CHAVE: Sarcopenia; Diálise peritoneal; Falência renal crônica.

\section{SARCOPENIA AND ASSOCIATED FACTORS IN PATIENTS WITH CHRONIC KIDNEY DISEASE IN PERITONEAL DI- ALYSIS TREATMENT}

ABSTRACT: Sarcopenia and other associated factors in patients with Chronic Kidney Disease (RKD) are evaluated. Patients underwent peritoneal dialysis (PD) in a nephrology clinic and were evaluated by a transversal study with PD users of both sexes. Sociodemographic, clinical conditions, hemodynamic and anthropometric data were retrieved of all individuals $(\mathrm{n}=35)$, with PD in the service under analysis. Sarcopenia was detected in $100 \%(n=14)$ of males. Undernourished, measured by muscular area and perimeter of arm, $78.6 \%(n=11)$ and $71.4 \%(n=10)$ were sarcopenic, as most of the hypertensive patients $57.1 \%(n=8)$. Sarcopenia was also positively associated with hypertriglyceridemia. Factors, such as gender, race/color, morbidity-based secondary diseases, arterial pressure and parameters of the nutritional status were associated with sarcopenia in the population studied.

KEY WORDS: Sarcopenia; Peritoneal dialysis; Kidney Failure, Chronic. 


\section{INTRODUÇÃO}

A Doença Renal Crônica (DRC) tem aumentado em todo o mundo e recebido cada vez mais atenção da comunidade científica $^{1-2}$. No Brasil, dados do Censo de 2016, realizado pela Sociedade Brasileira de Nefrologia (SBN), demonstraram que o total de pacientes em tratamento dialítico era de 100.397 em 2013 e de 122.825 em 2016, o que representa aumento de 22,33\%, sendo que destes, $83 \%$ tinham como fonte pagadora o Sistema Único de Saúde (SUS) ${ }^{3}$.

Nesse cenário, a Diálise Peritoneal (DP) é uma importante modalidade de terapia renal substitutiva, principalmente nos estágios finais da DRC . Segundo o censo da SBN de $2016^{3}$, há 2.951 pessoas em tratamento substitutivo do tipo diálise peritoneal automática, sendo este número também superior ao do censo de $2013^{5}$.

Ressalta-se que restrições dietéticas e hídricas e constante inapetência, assim como infecções, sangramentos e espoliações hidroeletrolíticas ${ }^{6,7}$ encontradas em pacientes em DP, podem desencadear piora no quadro nutricional e de saúde geral do paciente, acarretando perda de proteínas, aminoácidos e vitaminas hidrossolúveis, favorecendo perda de massa muscular e de força progressiva, o que caracteriza a sarcopenia ${ }^{8-10}$.

Esta perda de massa muscular ocorre em todas as etapas da DRC e quanto maior a perda da função renal, maior o risco de apresentar sarcopenia ${ }^{10}$. A etiologia da perda de massa muscular que ocorre na DRC é multifatorial e, atualmente, recebe o nome de sarcopenia urêmica ${ }^{11}$, obtendo diversas causas específicas, entre elas causas hormonais, alterações imunológicas, acidose metabólica, redução na ingesta proteica, inatividade física e excesso de angiotensina $\mathrm{II}^{10}$. A presença de sarcopenia pode estar ligada ao maior comprometimento da qualidade de vida, à maior facilidade de quedas e lesões ${ }^{12}$, ao aumento nos custos com o tratamento e à hospitalização recorrente ${ }^{10,13}$, entretanto estudos que investigam esta condição em pacientes em DP são escassos. Dessa forma, este estudo tem como objetivo avaliar a presença de sarcopenia e fatores associados em pacientes com DRC que realizaram DP em ambulatório de nefrologia num hospital universitário.

\section{METODOLOGIA}

Trata-se de um estudo quantitativo, observacional, analítico e transversal, no qual fizeram parte $100 \%$ $(\mathrm{n}=35)$ dos pacientes atendidos em ambulatório de nefrologia de um Hospital Universitário da Grande Vitória, Espírito Santo, Brasil. Foram incluídos pacientes com 20 anos ou mais, de ambos os sexos e que realizavam terapia renal substitutiva do tipo DP por seis meses ou mais.

Cada paciente foi abordado durante sua visita mensal ao hospital, no momento em que realizava seu atendimento de rotina com médico e nutricionista. Os participantes realizavam DP do tipo automática noturna, portanto, nenhum possuía abdome distendido em decorrência do dialisato, visto que este é retirado por completo pela máquina cicladora. Inicialmente, o paciente respondeu a um questionário semiestruturado sobre características sociodemográficas e história familiar e clínica. Posteriormente, foram coletados dados bioquímicos no prontuário do paciente, além de dados clínicos.

Foram avaliadas como características sociodemográficas o sexo, faixa etária categorizada em "20 a 40 anos", "41 a 60 anos" e "mais de 60 anos", raça/cor autorreferida, estado civil, escolaridade, profissão, número de pessoas que vivem na residência e a classe socioeconômica estabelecida de acordo com o critério de classificação econômica do Brasil ${ }^{14}$ e categorizada em "B" e "C" e "D + E", visto que nenhum indivíduo analisado estava inserido na classe socioeconômica A.

As variáveis do estilo de vida e história clínica do paciente incluíram tempo de DP, se o paciente realizou hemodiálise anterior, comorbidades associadas e doenças de base.

Para a análise bioquímica foram coletados os valores dos exames mais recentes e mais próximos da realização da coleta no prontuário do paciente, e classificados de acordo com a referência específica para pacientes dialíticos, segundo Riella e Martins ${ }^{15}$. Foram coletados o colesterol total, os triglicerídeos, a glicemia, o hematócrito, a hemoglobina, o ferro sérico, a ferritina, o fósforo, o cálcio total, o paratormônio, o sódio, o potássio, a ureia, a creatinina, as proteínas totais e a albumina.

Os dados hemodinâmicos coletados foram as medidas da pressão arterial sistólica (PAS) e pressão arte- 
rial diastólica $(\mathrm{PAD})$. Estas medidas foram aferidas durante a entrevista, utilizando aparelho automático de braço devidamente calibrado e validado pelo Instituto Nacional de Metrologia, Qualidade e Tecnologia (INMETRO). Foram seguidos todos os procedimentos e a classificação foi descrita no protocolo VII Diretrizes Brasileiras de Hipertensão $0^{16}$.

O estado nutricional foi avaliado por meio dos seguintes parâmetros antropométricos: peso corporal (kg), medido em balança mecânica da marca Micheletti ${ }^{\circledR}$ (São Paulo, Brasil), com capacidade de até $150 \mathrm{~kg}$ do tipo plataforma; estatura (m), aferida por meio de estadiômetro portátil da marca AlturaExata ${ }^{\circledR}$ (Belo Horizonte, Brasil), com escala bilateral em milímetros e capacidade de uso de 0,35 a 2,13 m; perímetro do braço (PB), aferido com fita inextensível da marca Cescorf® (Paraná, Brasil). Por fim, as pregas cutâneas tricipital (PCT), bicipital (PCB), subescapular (PCSE) e suprailíaca (PCSI) (mm) foram aferidas com o adipômetro do tipo Lange Skinfold Caliper (Cambridge Scientific Industries Inc.), utilizando-se o valor médio de três medidas.

Foi realizado o cálculo do Índice de Massa Corporal (IMC $=\mathrm{kg} / \mathrm{m}^{2}$ ), classificando-o segundo a Organização Mundial da Saúde (OMS) ${ }^{17}$. A fim de ter uma classificação mais fidedigna do peso corporal seco dos participantes, foi realizado um desconto de peso em caso de presença de edema, conforme a escala de cruzes proposta por Martins ${ }^{18}$.

A partir dos valores encontrados nas PCT dos indivíduos e de $\mathrm{PB}$, foi realizada a adequação da PCT e adequação de $\mathrm{PB}$, respectivamente, segundo a fórmula desenvolvida por Blacknurn e Thornton ${ }^{19}$. Portanto, para a classificação de indivíduos em sarcopênicos ou não sarcopênicos, foi utilizada a quantidade de Massa Livre de Gordura Apendicular (membros superiores e membros inferiores - MLGA), calculada pela fórmula MLGA = $-14,529+(17,989$ x estatura em metros $)+0,1307 \times$ MG total kg/ (estatura) ${ }^{2}$, classificando-se em sarcopênicos indivíduos que possuíam o valor inferior a $7,26 \mathrm{~kg} / \mathrm{m}^{2}$ para homens e $5,45 \mathrm{~kg} / \mathrm{m}^{2}$ para mulheres 9 .

Para a classificação da reserva de gordura corporal, utilizada na fórmula acima, foi calculado o percentual de gordura corporal a partir do somatório das quatro pregas cutâneas bicipital, tricipital, subescapular e su- prailíaca, no qual o resultado foi empregado na equação de Durnin e Womersely ${ }^{20}$ e a classificação feita segundo Lohman ${ }^{21}$. Foi realizada, também, a correção da área óssea por meio do cálculo da área muscular do braço corrigida (AMBc) e para sua classificação utilizaram-se as tabelas elaboradas por Frisancho ${ }^{22}$.

Foram utilizadas medidas de tendência central (média e mediana) e medidas de dispersão (desvios-padrão e intervalo interquartílico) para as variáveis contínuas conforme a normalidade das variáveis, identificado pelo teste de Kolmogorov-Smirnov. Já para as variáveis categóricas, utilizaram-se medidas percentuais. Para a análise das diferenças das proporções foi utilizado o teste Qui-quadrado $\left(\mathrm{X}^{2}\right)$ e o teste Exato de Fisher. O nível de significância adotado foi de $\mathrm{p}<0,05$. As análises estatísticas foram realizadas utilizando-se o software IBM SPSS Statistics 22.0.

O trabalho foi aprovado pelo Comitê de Ética em Pesquisa (CEP) da Universidade Federal do Espírito Santo (UFES), sob o número CAAE 52744515.0.0000.5060 (parecer 1.471.364). A participação dos indivíduos foi de forma voluntária e o consentimento foi dado por escrito por meio da assinatura do Termo de Consentimento Livre e Esclarecido (TCLE).

\section{RESULTADOS}

Foram avaliados $100 \%(n=35)$ dos pacientes com no mínimo seis meses de DP atendidos em um Hospital Universitário no período da coleta. A média de idade dos participantes foi de 54,4 ( $\pm 18,7)$ anos, sendo que a maioria dos dialíticos era do sexo feminino $(57,14 \%)$, aposentados ou pensionistas (60\%), da classe socioeconômica C (62,85\%), moravam com o(a) companheiro(a) $(57,14 \%)$, eram negros ou pardos $(71,42 \%)$ e possuía menos de quatro anos de estudo (45,71\%). Com relação à história clínica, 31,42\% dos indivíduos apresentavam até duas comorbidades associadas à DRC e 40\% não haviam realizado tratamento hemodialítico anterior à DP. A respeito da adequação do $\mathrm{PB}$ e da $\mathrm{AMBc}$, constatou-se que os homens eram mais comumente classificados como desnutridos $(66,7 \%, \mathrm{n}=10$ para $\mathrm{PB}$ e $78,6 \%, \mathrm{n}=11$ para $A M B c)$ e hipertensos $(53,3 \%)$ (Tabela 1$)$. 
Quando a sarcopenia foi analisada com as variáveis sociodemográficas (Tabela 2), foi demonstrado que $100 \%$ dos homens foram classificados como sarcopênicos. Nenhuma mulher analisada, no entanto, foi igualmente classificada. Encontrou-se, ainda, que 90\% dos negros e pardos não possuíam sarcopenia, diferentemente dos brancos, entre os quais $50 \%$ foram classificados como sarcopênicos.

Os indivíduos com doença renal secundária à comorbidades apresentaram menor taxa de sarcopenia (80\%), classificados, então, como não sarcopênicos. Nenhuma outra variável associou-se com a sarcopenia nesta categoria.

Ao se realizar a análise do desfecho do estudo com as variáveis antropométricas e hemodinâmicas dos participantes (Tabela 4), a maior parte dos indivíduos desnutridos, segundo a adequação do PB $(71,4 \%)$ e segundo a $\mathrm{AMBc}(78,36 \%)$, foram classificados como sarcopênicos. Com relação à PA, observou-se que 57,1\% dos indivíduos classificados como hipertensos foram também classificados como sarcopênicos.

De acordo com a análise das médias/medianas das variáveis bioquímicas em relação à sarcopenia (Tabela 5), observou-se que os indivíduos sarcopênicos possuíam maiores médias de triglicerídeos ao se comparar com indivíduos não sarcopênicos.

\section{DISCUSSÃO}

Os principais achados deste estudo demonstraram que houve presença de sarcopenia em todos os indivíduos do sexo masculino investigados, sendo estes mais comumente classificados como desnutridos. Em um estudo com idosos, realizado por Landi et al. ${ }^{23}$, também foi evidenciada a maior prevalência de sarcopenia no sexo masculino do que no feminino, devido a maior presença de gordura corporal isto corrobora com estudos já realizados tanto no Brasil quanto internacionalmente, nos quais se constatou que as mulheres tendem a ter maior acúmulo de gordura subcutânea do que os indivíduos do sexo masculino ${ }^{24-25}$.

Com relação à presença de sarcopenia na população considerada negra e parda, Volpato et al ${ }^{26}$ verificaram que na população brasileira não quilombola a presença de sarcopenia foi maior, mesmo quando observadas faixas etárias com idades inferiores. Além disso, Jones et al. ${ }^{27}$ realizaram um estudo no qual constataram que mulheres de etnia negra possuíam maior quantidade de massa muscular esquelética do que as de etnia branca de idade, peso e estatura semelhantes, corroborando, assim, com o presente estudo.

As doenças de base secundárias às comorbidades, como diabetes e hipertensão arterial, foram inversamente associados à sarcopenia, resultado diferente do encontrado por outro estudo no qual estas comorbidades pareceram contribuir para o declínio da massa muscular ${ }^{28}$.

Alguns achados, no entanto, qualificam a presença de sarcopenia como causadora de efeitos deletérios à saúde, sobretudo da população idosa ${ }^{29}$, entre os quais se destacam: desordens cardiometabólicas ${ }^{30}$, síndrome metabólica ${ }^{31}$, diabetes mellitus ${ }^{32}$ e doença arterial coronaria$n a^{33}$.

A respeito dos valores encontrados na adequação do $\mathrm{PB}$ e da $\mathrm{AMBc}$, os indivíduos que possuíam maior desnutrição foram classificados como sarcopênicos com mais frequência. Tais resultados evidenciam a eficácia destes instrumentos em detectar a presença ou predisposição à sarcopenia, levando em consideração a classificação do estado nutricional do indivíduo. Outros estudos também evidenciaram estes parâmetros como bons indicativos de reserva de massa muscular, o que valida sua utilização em estudos antropométricos ${ }^{34-35}$.

A respeito dos dados bioquímicos encontrados, somente o triglicerídeo associou-se à sarcopenia. Convém ressaltar que pacientes com DRC geralmente possuem hipertrigliceridemia ${ }^{36}$, sendo este fator considerado também de morbimortalidade aliado à desnutrição, anemia $\mathrm{e}$ ao processo inflamatório existentes no grupo estudado ${ }^{37}$.

Encontrou-se, ainda, que além dos indivíduos desnutridos, os eutróficos, com sobrepeso e com obesidade também puderam ser classificados como sarcopênicos. Na prática clínica, isto dificulta uma ação eficaz sobre o tratamento da sarcopenia, visto que esta pode ser mascarada pela grande quantidade de massa gorda, condição esta denominada obesidade sarcopênica ${ }^{8}$. Esta última, muitas vezes, está relacionada a disfunções físicas se comparada a situaçóes em que somente a sarcope- 
nia ou somente a obesidade estivesse presente. Por este fato, Newman et al..$^{38}$ destacaram recentemente o quão importante a massa gorda é ao se considerar a presença de sarcopenia, visto que indivíduos com peso corporal normal, muitas vezes, podem não ser classificados como sarcopênicos.

Importa ressaltar que este estudo apresenta algumas limitações. Em primeiro lugar, a natureza transversal da pesquisa não permite indicar uma relação de causa efeito a respeito da sarcopenia. No entanto, a associação realizada nos traz um panorama da presente situação de saúde dos pacientes analisados. Além disso, os pontos de corte e os métodos para detecção de sarcopenia na população analisada devem ser melhor estabelecidos, visto as características peculiares dos pacientes com DRC. Apesar do número reduzido de participantes do presente estudo, ressalta-se que este representa a população, ou seja, $100 \%$ dos indivíduos em DP atendidos neste serviço de nefrologia no período da coleta de dados. Esse fato é comumente encontrado em outros estudos dessa natureza, visto que a população que realiza DP normalmente é menor nas clínicas de diálise 3 . Dessa forma, sugerem-se novos estudos que englobem dados multicêntricos a fim de traçar um panorama da condição de saúde de pacientes em DP de forma mais abrangente.

\section{CONCLUSÃO}

Conclui-se, portanto, que a sarcopenia está presente em pacientes que realizam DP, sendo que fatores como sexo, cor, doenças de base secundárias a cormobidades, pressão arterial e determinados parâmetros de estado nutricional tiveram associação com a presença de sarcopenia na população estudada.

Tais achados ressaltam a importância da análise da presença desta perda de massa muscular em indivíduos com DRC, caracterizando-se como uma condição determinante para a qualidade de vida do paciente, para a diminuição dos custos com o tratamento e para o aumento da sobrevida destes indivíduos, além de compreender os mecanismos desencadeadores desta condição, tais como sedentarismo ou alimentação deficiente em quantidade proteica. Por fim, entender a situação do estado nutricional dos pacientes em diálise pode levar à adoção de medidas preventivas e terapêuticas mais eficazes nes- tas situações.

\section{AGRADECIMENTOS}

Os autores agradecem ao setor de Nefrologia do Hospital Universitário de Vitória/ES/BR, pela parceria na pesquisa e aos pacientes do setor de diálise peritoneal pela disponibilidade.

\section{REFERÊNCIAS}

1. Bastos MG, Kirsztajn GM. Doença renal crônica: importância do diagnóstico precoce, encaminhamento imediato e abordagem interdisciplinar estruturada para melhora do desfecho em pacientes ainda não submetidos à diálise. J Bras Nefrol 2011;33(1):93108.

2. Malta DC, Berna RTI, Lima MG, Araújo SSC, Silva MMA, Freitas MIF, et al. Doenças crônicas não transmissíveis e a utilização de serviços de saúde: análise da Pesquisa Nacional de Saúde no Brasil. Rev Saude Publica 2017; 51(Supl 1):10-11

3. Sociedade Brasileira de Nefrologia. Censo da Sociedade Brasileira de Nefrologia. 2016. Disponível em: http://www.sbn.org.br/

4. Najafi I, Ossareh S, Hosseini M, Ganji MR, Naghibi M, Makhdoomi K, et al. Epidemiology of culture- negative peritonitis in irarian patients on continuous ambulatory peritoneal dialysis. Jour Of Kidney Diseases 2011; 5(5): 332-7.

5. Sociedade Brasileira de Nefrologia. Censo da Sociedade Brasileira de Nefrologia. 2013. Disponível em: http://www.sbn.org.br/

6. Duarte EAC, Silva GA, Dias RC, Carvalho CG. Nutricional Evaluation of peritoneal dialysis patients in miner Institute of Nephrology of Belo Horizonte (MG). e-Scientia 2012;5(2):24-32.

7. Kusumota L, Rodrigues RAP, Marques S. Idosos com insuficiência renal crônica: alterações do estado de saúde. Rev Latino-Am Enfermagem 2004;12(3):52532

8. Silva Neto LS, Karnikowiski MGO, Tavares AB, Lima RM. Associação entre sarcopenia, obesidade sarcopênica e força muscular com variáveis relacionadas 
de qualidade de vida em idosas. Rev. bras. fisioter 2012;16(5):360-367.

9. Baumgartner RN, Koehler KM, Gallagher D, Romero L, Heymsfield SB, Ross RR et al. Epidemiology of sarcopenia among the elderly in New Mexico. Am J Epidemiol 1998;147(8):755-63.

10. Souza VA, Oliveira D, Mansur HN, Fernandes NMS, Bastos MG. Sarcopenia na Doença Renal Crônica. Jornal Brasileiro de Nefrologia 2015;37(1):98-105.

11. Fahal IH. Uremic sarcopenia: etiology and implications. Nephrol Dial Transplant 2014;29:1655-65.

12. Martinez BP, Camelier FWR, Camelier AA. Sarcopenia em idosos - um estudo de revisão. Rev Pesq Fisio 2014;4(1):62-70.

13. Silva TAA, Junior AF, Pinheiro MM, Szejnfeld VL. Sarcopenia Associada ao Envelhecimento: Aspectos Etiológicos e Opções Terapêuticas. Rev Bras Reumatol 2006;46(6):391-7.

14. ABEP - Associação Brasileira de Empresas de Pesquisa 2014. Disponível em: www.abep.org .

15. Riella MC, Martins C. Nutrição e o rim. 2. ed. Rio de Janeiro: Guanabara; 2013. p. 381.

16. Sociedade Brasileira de Cardiologia. Sociedade Brasileira de Hipertensão. Sociedade Brasileira de Nefrologia. VII Diretrizes Brasileiras de Hipertensão. Arq. Bras. Cardiol 2016;107(3):1-83.

17. World Health Organization (WHO). Physical Status: The use and interpretation of anthropometry. Geneva: World Health Organization (WHO); 2000.

18. Martins C, Cardoso SP. Terapia nutricional enteral e parenteral. Nutro Clínica. 2000.

19. Blackburn GL, Thornton, PA. Nutritional assessment of the hospitalized patient. Medical Clinics of North America 1979;14:1102-08.

20. Cuppari L. Guia de nutrição: nutrição clínica no adulto. 2. ed. Barueri, SP: Manole; 2005. p.474.

21. Lohman TG, Roche A F, Martorell R. Anthropometric standardization reference manual. Champaign: Human Kinetics; 1988.
22. Frisancho AR. Anthropmetric standarts for the assessment of growth and nutrition status. Michigan: The University of Michigan Press; 1990. p.189.

23. Landi F, Liperoti R, Fusco D, Mastropaolo S, Quattrociocchi S, Proia A, et al. Prevalence and Risk Factors of Sarcopenia Among Nursing Home Older Residents. J Gerontol A Biol Sci Med Sci 2012.67A(1):48-55.

24. Rosa EC, Zanella MT, Ribeiro AB, Junior OK. Obesidade Visceral, Hipertensão Arterial e Risco Cardio-renal: uma revisão. Arq Bras Endocrinol Metab. 2005; 49(2):196-204.

25. Colombo RCR, Aguillar OM, Gallani MCBJ, Gobatto CA. Caracterização da obesidade em pacientes com infarto do miocárdio. Rev Latino-am Enfermagem 2003; 11(4):461-7

26. Volpato S, Bianchi L, Cherubini A, Landi F, Maggio M, Sa-vino E, et al. Prevalence and clinical correlates of sarcopenia in community-dwelling older people: application of the EWGSOP definition and diagnostic algorithm. J Gerontol A Biol Sci Med Sci 2014;69(4):438-46.

27. Jones A Jr, Shen W, St-Onge MP, Gallagher D, Heshka S, Wang Z, et al. Body-composition differences between African American and white women: relation to resting energy requirements. Am J Clin Nutr 2004;79(5):780-6.

28. Salmaso FV, Vigário PS, Mendonça LMC, Madeira M, Netto LV, Guimarães MRM, et al. Análise de idosos ambulatoriais quanto ao estado nutricional, sarcopenia, função renal e densidade óssea. Arq Bras Endocrinol Metab. 2014;58(3):226-31.

29. Janssen I, Shepard DS, Katzmarzyk PT, Roubenoff MDR. The Healthcare Costs of Sarcopenia in the United States. J Am Soc Geriatr. 2004;52(1):80-5.

30. Abellan Van Kan, G. Epidemiology and consequences of sarcopenia. The Journal of Nutrition, Health \& Aging. 2009;13(8):708-12.

31. Ishii S, Tanaka T, Akishita M, Ouchi Y, Tuji T, Iijima K. Metabolic Syndrome, Sarcopenia and Role of Sex and Age: Cross-Sectional Analysis of Kashiwa Cohort Study. 2014;9(11):e112718.

32. Kim KS, Park KS, Kim MJ, Kim SK, Cho YW, Park SW. 
Type 2 diabetes is associated with low muscle mass in older adults: Low muscle mass in older adults with type 2 diabetes. Geriatrics \& Gerontology International 2014;14:115-121.

33. Chin SO, Rhee SY, Chon S, Hwang YC, Jeong IK, Oh $S$, et al. Sarcopenia Is Independently Associated with Cardiovascular Disease in Older Korean Adults: The Korea National Health and Nutrition Examination Survey (KNHANES) from 2009. PLoS ONE 2013;8(3):e60119.

34. Barbosa RB, Souza JM, Lebrão ML, Marucci Mde FN. Anthropometry of elderly residents in the city of São Paulo, Brazil. Cad Saúde Pública 2005;21(6):1929-38.

35. Coqueiro RDAS, Barbosa AR, Borgatto AF. Anthropometric measurements in the elderly of Havana, Cuba: age and sex differences. Nutrition 2009;25(1):33-9.
36. Marques FS, da Silva ALM, Couto RD. Dislipidemia associada à doença renal crônica-Revisão de literatura. Revista de Ciências Médicas e Biológicas. 2015;13(2):220-5.

37. Ferreira SRC, Rocha AM, Saraiva JFK. Estatinas na doença renal crônica. Arquivos Brasileiros de Cardiologia 2005:85(5):45-9.

38. Newman AB, Kupelian V, Visser M, Simonsick E, Goodpaster B, Nevitt M, et al. Sarcopenia: alternative definitions and associations with lower extremity function. J Am Geriatr.Soc 2003;51(11):1602-09.

Recebido em: 14/08/2018 Aceito em: 04/09/2018

\section{ANEXOS}

Tabela 1. Caracterização de pacientes em tratamento dialítico peritoneal segundo sexo, conforme variáveis da história clínica e estado nutricional. Vitória (ES) Brasil, 2017

(Continua)

\begin{tabular}{|c|c|c|c|c|c|c|c|}
\hline \multirow{3}{*}{ Variáveis } & \multicolumn{4}{|c|}{ Sexo } & \multirow{2}{*}{\multicolumn{2}{|c|}{ Total }} & \multirow{3}{*}{ p valor } \\
\hline & \multicolumn{2}{|c|}{ Feminino } & \multicolumn{2}{|c|}{ Masculino } & & & \\
\hline & $\mathbf{N}$ & $\%$ & $\mathbf{N}$ & $\%$ & $\mathbf{N}$ & $\%$ & \\
\hline \multicolumn{8}{|l|}{ Tempo de DP } \\
\hline 3 a 6 meses & 5 & $25,0 \%$ & 3 & $20,0 \%$ & 8 & 22,9 & \multirow{4}{*}{0,500} \\
\hline 7 a 12 meses & 4 & $20,0 \%$ & 1 & $6,7 \%$ & 5 & 14,3 & \\
\hline 1 a 2 anos & 5 & $25,0 \%$ & 7 & $46,7 \%$ & 12 & 34,3 & \\
\hline 2 anos ou mais & 6 & $30,0 \%$ & 4 & $26,7 \%$ & 10 & 28,6 & \\
\hline \multicolumn{7}{|l|}{ HD anterior (meses) } & \multirow{5}{*}{0,401} \\
\hline Nunca & 8 & $40,0 \%$ & 6 & $40,0 \%$ & 14 & 40,0 & \\
\hline 1 a 3 meses & 3 & $15,0 \%$ & 5 & $33,3 \%$ & 8 & 22,9 & \\
\hline 4 a 6 meses & 5 & $25,0 \%$ & 1 & $6,7 \%$ & 6 & 17,1 & \\
\hline 6 meses ou mais & 4 & $20,0 \%$ & 3 & $20,0 \%$ & 7 & 20,0 & \\
\hline \multicolumn{7}{|l|}{ Comorbidades } & \multirow{4}{*}{0,975} \\
\hline Uma comorbidade & 10 & $50,0 \%$ & 7 & $46,7 \%$ & 17 & 48,6 & \\
\hline Duas comorbidades & 6 & $30,0 \%$ & 5 & $33,3 \%$ & 11 & 31,4 & \\
\hline Três ou mais comorbidades & 4 & $20,0 \%$ & 3 & $20,0 \%$ & 7 & 20,0 & \\
\hline \multicolumn{7}{|l|}{ Doença de base } & \multirow{5}{*}{0,051} \\
\hline Doença renal secundária à comorbidade & 16 & $80,0 \%$ & 8 & $53,3 \%$ & 24 & 68,6 & \\
\hline Doenças glomerulares & 4 & $20,0 \%$ & 2 & $13,3 \%$ & 6 & 17,1 & \\
\hline Doenças nefríticas & 0 & $0,0 \%$ & 2 & $13,3 \%$ & 2 & 5,7 & \\
\hline Outros & 0 & $0,0 \%$ & 3 & $20,0 \%$ & 3 & 8,6 & \\
\hline
\end{tabular}


(Conclusão)

\begin{tabular}{|c|c|c|c|c|c|c|c|}
\hline \multirow{3}{*}{ Variáveis } & \multicolumn{4}{|c|}{ Sexo } & \multirow{2}{*}{\multicolumn{2}{|c|}{ Total }} & \multirow{3}{*}{$p$ valor } \\
\hline & \multicolumn{2}{|c|}{ Feminino } & \multicolumn{2}{|c|}{ Masculino } & & & \\
\hline & $\mathbf{N}$ & $\%$ & $\mathbf{N}$ & $\%$ & $\mathbf{N}$ & $\%$ & \\
\hline \multicolumn{8}{|l|}{ Classificação IMC* } \\
\hline Baixo peso/eutrofia & 13 & $65,0 \%$ & 11 & $73,3 \%$ & 24 & 68,6 & \multirow[t]{2}{*}{0,721} \\
\hline Sobrepeso/obesidade & 7 & $35,0 \%$ & 4 & $26,7 \%$ & 11 & 31,4 & \\
\hline \multicolumn{7}{|l|}{ Adequação de PB } & \multirow{4}{*}{0,006} \\
\hline Desnutrição & 3 & $15,0 \%$ & 10 & $66,7 \%$ & 13 & 37,1 & \\
\hline Eutrofia & 11 & $55,0 \%$ & 4 & $26,7 \%$ & 15 & 42,9 & \\
\hline Excesso de peso & 6 & $30,0 \%$ & 1 & $6,7 \%$ & 7 & 20,0 & \\
\hline \multicolumn{7}{|l|}{ AMBc $^{1}$} & \multirow{4}{*}{0,001} \\
\hline Desnutrição & 0 & $0,0 \%$ & 11 & $78,6 \%$ & 11 & 32,4 & \\
\hline Eutrofia & 14 & $70,0 \%$ & 3 & $21,4 \%$ & 17 & 50,0 & \\
\hline Excesso de peso & 6 & $30,0 \%$ & 0 & $0,0 \%$ & 6 & 17,6 & \\
\hline \multicolumn{7}{|l|}{ Adequação PCT'1 } & \multirow{4}{*}{0,351} \\
\hline Desnutrição & 4 & $20,0 \%$ & 1 & $7,1 \%$ & 5 & 14,7 & \\
\hline Eutrofia & 5 & $25,0 \%$ & 2 & $14,3 \%$ & 7 & 20,6 & \\
\hline Excesso de peso & 11 & $55,0 \%$ & 11 & $78,6 \%$ & 22 & 64,7 & \\
\hline \multicolumn{7}{|l|}{ Pressão arterial } & \multirow{4}{*}{0,003} \\
\hline Normal & 0 & $0,0 \%$ & 5 & $33,3 \%$ & 5 & 14,3 & \\
\hline Pré-hipertenso & 12 & $60,0 \%$ & 2 & $13,3 \%$ & 14 & 40,0 & \\
\hline Hipertenso & 8 & $40,0 \%$ & 8 & $53,3 \%$ & 16 & 45,7 & \\
\hline
\end{tabular}

Tabela 2. Associação da sarcopenia segundo variáveis sociodemográficas de pacientes em Diálise Peritoneal. Vitória (ES) Brasil, 2017

(Continua)

\begin{tabular}{|c|c|c|c|c|c|c|c|}
\hline \multirow{3}{*}{ Variável } & \multicolumn{4}{|c|}{ Sarcopenia } & \multirow{2}{*}{\multicolumn{2}{|c|}{ Total }} & \multirow{3}{*}{$\mathrm{p}$ valor } \\
\hline & \multicolumn{2}{|c|}{ Sarcopênico } & \multicolumn{2}{|c|}{ Não sarcopênico } & & & \\
\hline & $\mathbf{N}$ & $\%$ & $\mathbf{N}$ & $\%$ & $\mathbf{N}$ & $\%$ & \\
\hline Sexo* & & & & & & & \multirow{3}{*}{0,001} \\
\hline Feminino & 0 & $0,0 \%$ & 20 & $100,0 \%$ & 20 & 58,8 & \\
\hline Masculino & 14 & $100,0 \%$ & 0 & $0,0 \%$ & 14 & 41,2 & \\
\hline \multicolumn{7}{|l|}{ Faixa etária } & \multirow{4}{*}{0,246} \\
\hline 20 a 40 anos & 6 & $42,9 \%$ & 4 & $20,0 \%$ & 10 & 29,4 & \\
\hline 41 a 60 anos & 2 & $14,3 \%$ & 7 & $35,0 \%$ & 9 & 26,5 & \\
\hline Mais de 60 anos & 6 & $42,9 \%$ & 9 & $45,0 \%$ & 15 & 44,1 & \\
\hline \multicolumn{7}{|l|}{ Raça/Cor } & \multirow{4}{*}{0,008} \\
\hline Branco & 7 & $50,0 \%$ & 1 & $5,0 \%$ & 8 & 23,5 & \\
\hline Amarelo e indígena & 1 & $7,1 \%$ & 1 & $5,0 \%$ & 2 & 5,9 & \\
\hline Preto e pardo & 6 & $42,9 \%$ & 18 & $90,0 \%$ & 24 & 70,6 & \\
\hline \multicolumn{7}{|l|}{ Estado civil* } & \multirow{3}{*}{0,171} \\
\hline Não mora com companheiro(a) & 4 & $28,6 \%$ & 11 & $55,0 \%$ & 15 & 44,1 & \\
\hline Mora com companheiro(a) & 10 & $71,4 \%$ & 9 & $45,0 \%$ & 19 & 55,9 & \\
\hline
\end{tabular}


(Conclusão)

\begin{tabular}{|c|c|c|c|c|c|c|c|}
\hline \multirow{3}{*}{ Variável } & \multicolumn{4}{|c|}{ Sarcopenia } & \multirow{2}{*}{\multicolumn{2}{|c|}{ Total }} & \multirow{3}{*}{ p valor } \\
\hline & \multicolumn{2}{|c|}{ Sarcopênico } & \multicolumn{2}{|c|}{ Não sarcopênico } & & & \\
\hline & $\mathbf{N}$ & $\%$ & $\mathbf{N}$ & $\%$ & $\mathbf{N}$ & $\%$ & \\
\hline Escolaridade & & & & & & & \multirow{5}{*}{0,320} \\
\hline Menos de 4 anos & 5 & $35,7 \%$ & 10 & $50,0 \%$ & 15 & 44,1 & \\
\hline 4 a 8 anos & 6 & $42,9 \%$ & 9 & $45,0 \%$ & 15 & 44,1 & \\
\hline 8 a 11 anos & 3 & $21,4 \%$ & 1 & $5,0 \%$ & 4 & 11,8 & \\
\hline 11 anos ou mais & 0 & $0,0 \%$ & 0 & $0,0 \%$ & 0 & 0,0 & \\
\hline \multicolumn{7}{|l|}{ Classe socioeconômica } & \multirow{4}{*}{0,780} \\
\hline B & 4 & $28,6 \%$ & 5 & $25,0 \%$ & 9 & 26,5 & \\
\hline $\mathrm{C}$ & 9 & $64,3 \%$ & 12 & $60,0 \%$ & 21 & 61,8 & \\
\hline $\mathrm{D} / \mathrm{E}$ & 1 & $7,1 \%$ & 3 & $15,0 \%$ & 4 & 11,8 & \\
\hline \multicolumn{7}{|l|}{ Profissão } & \multirow{4}{*}{0,161} \\
\hline Aposentado/afastado & 7 & $50,0 \%$ & 13 & $65,0 \%$ & 20 & 58,8 & \\
\hline Desempregado & 1 & $7,1 \%$ & 4 & $20,0 \%$ & 5 & 14,7 & \\
\hline Empregado & 6 & $42,9 \%$ & 3 & $15,0 \%$ & 9 & 26,5 & \\
\hline \multicolumn{7}{|c|}{$\begin{array}{l}\text { Número de pessoas que residem na } \\
\text { casa }\end{array}$} & \multirow{5}{*}{0,681} \\
\hline Mora sozinho(a) & 3 & $21,4 \%$ & 4 & $20,0 \%$ & 7 & 20,6 & \\
\hline Duas pessoas & 6 & $42,9 \%$ & 5 & $25,0 \%$ & 11 & 32,4 & \\
\hline Três pessoas & 2 & $14,3 \%$ & 5 & $25,0 \%$ & 7 & 20,6 & \\
\hline$\geq$ quatro pessoas & 3 & $21,4 \%$ & 6 & $30,0 \%$ & 9 & 26,5 & \\
\hline
\end{tabular}

Fonte: Teste qui-quadrado. * Teste Exato de Fisher.

Nota: $\mathrm{N}=34$.

Tabela 3. Associação da sarcopenia segundo variáveis de estilo de vida e história clínica de pacientes em Diálise Peritoneal. Vitória (ES) Brasil, 2017

(Continua)

\begin{tabular}{|c|c|c|c|c|c|c|c|}
\hline \multirow{3}{*}{ Variável } & \multicolumn{4}{|c|}{ Sarcopenia } & \multirow{2}{*}{\multicolumn{2}{|c|}{ Total }} & \multirow{3}{*}{ p valor } \\
\hline & \multicolumn{2}{|c|}{ Sarcopênico } & \multicolumn{2}{|c|}{ Não sarcopênico } & & & \\
\hline & $\mathbf{N}$ & $\%$ & $\mathbf{N}$ & $\%$ & $\mathbf{N}$ & $\%$ & \\
\hline Tempo de DP & & & & & & & \multirow{5}{*}{0,416} \\
\hline 3 a 6 meses & 2 & $14,3 \%$ & 5 & $25,0 \%$ & 7 & 20,6 & \\
\hline 7 a 12 meses & 1 & $7,1 \%$ & 4 & $20,0 \%$ & 5 & 14,7 & \\
\hline 1 a 2 anos & 7 & $50,0 \%$ & 5 & $25,0 \%$ & 12 & 35,3 & \\
\hline 2 anos ou mais & 4 & $28,6 \%$ & 6 & $30,0 \%$ & 10 & 29,4 & \\
\hline \multicolumn{7}{|l|}{ HD anterior (meses) } & \multirow{5}{*}{0,368} \\
\hline Nunca & 6 & $42,9 \%$ & 8 & $40,0 \%$ & 14 & 41,2 & \\
\hline 1 a 3 meses & 5 & $35,7 \%$ & 3 & $15,0 \%$ & 8 & 23,5 & \\
\hline 4 a 6 meses & 1 & $7,1 \%$ & 5 & $25,0 \%$ & 6 & 17,6 & \\
\hline 6 meses ou mais & 2 & $14,3 \%$ & 4 & $20,0 \%$ & 6 & 17,6 & \\
\hline \multicolumn{7}{|l|}{ Cormobidades } & \multirow{4}{*}{0,889} \\
\hline Uma comorbidade & 7 & $50,0 \%$ & 10 & $50,0 \%$ & 17 & 50,0 & \\
\hline Duas comorbidades & 5 & $35,7 \%$ & 6 & $30,0 \%$ & 11 & 32,4 & \\
\hline Três ou mais comorbidades & 2 & $14,3 \%$ & 4 & $20,0 \%$ & 6 & 17,6 & \\
\hline
\end{tabular}


(Conclusão)

\begin{tabular}{|c|c|c|c|c|c|c|c|}
\hline \multirow{3}{*}{ Variável } & \multicolumn{4}{|c|}{ Sarcopenia } & \multirow{2}{*}{\multicolumn{2}{|c|}{ Total }} & \multirow{3}{*}{ p valor } \\
\hline & \multicolumn{2}{|c|}{ Sarcopênico } & \multicolumn{2}{|c|}{ Não sarcopênico } & & & \\
\hline & $\mathbf{N}$ & $\%$ & $\mathbf{N}$ & $\%$ & $\mathbf{N}$ & $\%$ & \\
\hline \multicolumn{7}{|l|}{ Doença de base } & \multirow{5}{*}{0,039} \\
\hline Doença renal secundária à comorbidade & 7 & $50,0 \%$ & 16 & $80,0 \%$ & 23 & 67,6 & \\
\hline Doenças glomerulares & 2 & $14,3 \%$ & 4 & $20,0 \%$ & 6 & 17,6 & \\
\hline Doenças nefríticas & 2 & $14,3 \%$ & 0 & $0,0 \%$ & 2 & 5,9 & \\
\hline Outros & 3 & $21,4 \%$ & 0 & $0,0 \%$ & 3 & 8,8 & \\
\hline
\end{tabular}

Fonte: Teste qui-quadrado. *Teste Exato de Fisher. $\mathrm{N}=34$.

Nota: DP: diálise peritoneal. HD: hemodiálise.

Tabela 4. Associação da sarcopenia segundo variáveis antropométricas e hemodinâmicas de pacientes em Diálise Peritoneal. Vitória (ES) Brasil, 2017

\begin{tabular}{|c|c|c|c|c|c|c|c|}
\hline \multirow{3}{*}{ Variável } & \multicolumn{4}{|c|}{ Sarcopenia } & \multirow{2}{*}{\multicolumn{2}{|c|}{ Total }} & \multirow{3}{*}{ p valor } \\
\hline & \multicolumn{2}{|c|}{ Sarcopênico } & \multicolumn{2}{|c|}{ Não sarcopênico } & & & \\
\hline & $\mathbf{N}$ & $\%$ & $\mathbf{N}$ & $\%$ & $\mathbf{N}$ & $\%$ & \\
\hline Classificação IMC* & & & & & & & \multirow{3}{*}{1,0} \\
\hline Baixo peso/eutrofia & 10 & $71,4 \%$ & 13 & $65,0 \%$ & 23 & 67,6 & \\
\hline Sobrepeso/obesidade & 4 & $28,6 \%$ & 7 & $35,0 \%$ & 11 & 32,4 & \\
\hline \multicolumn{7}{|l|}{ Adequação PB } & \multirow{4}{*}{0,004} \\
\hline Desnutrição & 10 & $71,4 \%$ & 3 & $15,0 \%$ & 13 & 38,2 & \\
\hline Eutrofia & 3 & $21,4 \%$ & 11 & $55,0 \%$ & 14 & 41,2 & \\
\hline Excesso de peso & 1 & $7,1 \%$ & 6 & $30,0 \%$ & 7 & 20,6 & \\
\hline \multicolumn{7}{|l|}{ AMBc } & \multirow{4}{*}{0,001} \\
\hline Desnutrição & 11 & $78,6 \%$ & 0 & $0,0 \%$ & 11 & 32,4 & \\
\hline Eutrofia & 3 & $21,4 \%$ & 14 & $70,0 \%$ & 17 & 50,0 & \\
\hline Excesso de peso & 0 & $0,0 \%$ & 6 & $30,0 \%$ & 6 & 17,6 & \\
\hline \multicolumn{7}{|l|}{ Adequação PCT } & \multirow{4}{*}{0,351} \\
\hline Desnutrição & 1 & $7,1 \%$ & 4 & $20,0 \%$ & 5 & 14,7 & \\
\hline Eutrofia & 2 & $14,3 \%$ & 5 & $25,0 \%$ & 7 & 20,6 & \\
\hline Excesso de peso & 11 & $78,6 \%$ & 11 & $55,0 \%$ & 22 & 64,7 & \\
\hline \multicolumn{7}{|l|}{ Pressão arterial } & \multirow{4}{*}{0,005} \\
\hline Normal & 4 & $28,6 \%$ & 0 & $0,0 \%$ & 4 & 11,8 & \\
\hline Pré-hipertenso & 2 & $14,3 \%$ & 12 & $60,0 \%$ & 14 & 41,2 & \\
\hline Hipertenso & 8 & $57,1 \%$ & 8 & $40,0 \%$ & 16 & 47,1 & \\
\hline
\end{tabular}

Fonte: Teste qui-quadrado. * Teste Exato de Fisher. $\mathrm{N}=34$.

Nota: PB: Perímetro do Braço; AMBc: Área Muscular do Braço corrigida; PCT: Prega Cutânea Tricipital. 
Tabela 5. Associação da sarcopenia segundo variáveis bioquímicas de pacientes em Diálise Peritoneal. Vitória (ES) Brasil, 2017

\begin{tabular}{|c|c|c|c|c|c|}
\hline \multirow{3}{*}{ Variáveis } & \multicolumn{4}{|c|}{ Classificação da sarcopenia } & \multirow{3}{*}{ p valor } \\
\hline & \multicolumn{2}{|c|}{ Sarcopênico } & \multicolumn{2}{|c|}{ Não sarcopênico } & \\
\hline & Média / Mediana & DP / IIQ & Média / Mediana & DP / IIQ & \\
\hline Colesterol total (Média \pm DP) & 164,4 & 60,6 & 194,9 & 54,1 & 0,133 \\
\hline 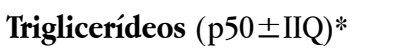 & 106,5 & 65,0 & 187,0 & 83,5 & 0,006 \\
\hline Glicemia $(\mathrm{p} 50 \pm \mathrm{IIQ})^{* 1}$ & 89,0 & 61,0 & 87,0 & 26,0 & 1 \\
\hline Hematócrito (Média $\pm D P)$ & 33,0 & 4,9 & 33,6 & 5,6 & 0,738 \\
\hline Hemoglobina (Média $\pm D P)$ & 11,1 & 1,6 & 10,9 & 1,6 & 0,621 \\
\hline Ferro sérico $(\mathrm{p} 50 \pm \mathrm{IIQ})^{* 2}$ & 68,0 & 43,0 & 63,5 & 34,0 & 0,332 \\
\hline Ferritina $(\mathrm{p} 50 \pm \mathrm{IIQ})^{* 3}$ & 211,0 & 260,9 & 218,0 & 348,4 & 0,97 \\
\hline Fósforo (Média $\pm D P$ ) & 5,4 & 1,1 & 5,2 & 1,2 & 0,596 \\
\hline Cálcio total (Média $\pm D P$ ) & 9,0 & 0,7 & 9,3 & 0,7 & 0,306 \\
\hline PTH $(\mathrm{p} 50 \pm \mathrm{IIQ})^{*}$ & 425,7 & 402,0 & 391,1 & 382,4 & 0,306 \\
\hline Sódio (Média $\pm D P$ ) & 138,1 & 3,2 & 138,1 & 3,2 & 0,985 \\
\hline Potássio (Média $\pm D P$ ) & 4,8 & 0,5 & 4,9 & 0,6 & 0,715 \\
\hline Ureia (Média $\pm D P$ ) & 103,2 & 28,9 & 108,0 & 36,9 & 0,688 \\
\hline Creatinina $(\text { Média } \pm D P)^{4}$ & 10,5 & 4,8 & 8,2 & 3,0 & 0,094 \\
\hline Proteínas totais $(\text { Média } \pm D P)^{4}$ & 6,3 & 0,7 & 6,1 & 0,8 & 0,414 \\
\hline Albumina (Média $\pm D P)$ & 3,7 & 0,3 & 3,7 & 0,5 & 0,973 \\
\hline
\end{tabular}

Fonte: Teste t de Student. * Teste U de Mann Whitney.

Nota: $\mathrm{N}=35 .{ }^{1} \mathrm{~N}=29 .{ }^{2} \mathrm{~N}=32 .{ }^{3} \mathrm{~N}=33 .{ }^{4} \mathrm{~N}=34$.

p50: mediana; DP: desvio padrão. IIQ: intervalo interquartílico. PTH: paratormônio. 